\title{
"Click" chemistry as a tool to create novel biomaterials: a short review
}

\author{
Mariana Barbosa ${ }^{1}$, Cristina Martins ${ }^{2}$, Paula Gomes ${ }^{3}$ \\ ${ }^{1}$ Department of Chemistry and Biochemistry, Faculty of Sciences University of Porto, Portugal \\ (mariana.barbosa@fc.up.pt); ${ }^{2} \mathrm{INEB}$ - Instituto de Engenharia Biomédica, University of Porto \\ (CMartins@ineb.up.pt); ${ }^{3}$ Department of Chemistry and Biochemistry, Faculty of Sciences \\ University of Porto, Portugal (pgomes@fc.up.pt)
}

\begin{abstract}
In recent years, there has been a growing demand for novel strategies for biomedical applications. Chitosan is a typical cationic amino-containing polysaccharide that has been widely used due to its unique properties. The grafting modification of chitosan has been explored as an interesting method to develop multifunctional novel chitosan hybrid materials for drug delivery, tissue engineering, and other biomedical applications. Recently, "click" chemistry has been introduced into the synthesis of polymeric materials with well-defined and complex chain architectures. The Huisgen's 1,3-dipolar cycloaddition reaction between alkynes and azides yielding triazoles is the principal example of a "click" reaction. Bioconjugation, surface modification, and orthogonal functionalization of polymers were successfully performed through this chemoselective reaction. In recent literature interest has been shown in this cycloaddition for the modification of polysaccharides, however, only a few chitosan graft copolymers have been synthesized by this technique.
\end{abstract}

Subject Headings. Chemical process, biomaterials, biotechnology, polymer. Author Keywords. Chitosan, chemoselective modification, Huisgen's 1,3-dipolar cycloaddition, biomaterials.

\section{Introduction}

In recent years, there has been a growing demand for novel strategies for biomedical applications. These strategies require biocompatible materials to serve as either structural support for tissue regeneration or delivery vehicles for cell and drug transplantation. This, however, is a challenging task due to the complexity of biological environment that impose the engineering of well-defined materials and surfaces (Nimmo and Shoichet 2011, Azagarsamy and Anseth 2012).

The recent progress in biotechnology and regenerative medicine has boosted the development of new synthetic organic techniques within regenerative biomaterials. In view of that, chemical conjugations are viewed as promising tools to not only to interrogate biomolecules in their native environment, but also to build materials for biomedical applications. However, most chemical reactions require long reaction times, strict exclusion of water, protection of the competing chemical groups, and vigorous heating/cooling or agitation. So, there has been a growing demand for engineering flexible, rapid, selective, and high yielding conjugation methods compatible with the complex biological milieu and physiological conditions (Nimmo and Shoichet 2011, Azagarsamy and Anseth 2012, Deng et al. 2011). These restricted and controlled reaction conditions were overcome with the advent of "click" chemistry. The term was introduced in 2001 by Sharpless et al. (Kolb et al. 2001) and 
comprises a set of chemical conjugations that resemble natural biochemical ligations (Nimmo and Shoichet 2011, Azagarsamy and Anseth 2012).

In this review paper, we give an overview on chitosan and its biomedical relevance, "click" reactions, namely the Huisgen's 1,3-dipolar cycloaddition, as a tool to chemically modify this polymer and finally the emerging biomedical applications of new functional chitosan derivatives developed following this synthetic approach.

\section{Chitosan, a natural biopolymer of biomedical relevance}

Chitosan is a linear, polycationic copolymer of $\beta$-(1 $\rightarrow 4)$-linked 2-acetamido-2-deoxy-Dglucopyranose and 2-amino-2-deoxy-D-glucopyranose units derived from the complete or partial $\mathrm{N}$-deacetylation of chitin, which is the main component of the exoskeleton of crustaceans as well as of the cell walls of some bacteria and fungi, widely available in nature (Tan et al. 2014, Pandis et al. 2014, Berger et al. 2004b, Elsabee and Abdou 2013, Costa et al. 2012, Zampano et al. 2010). Chitosan is characterized by its molecular weight and degree of deacetylation, representing the proportion of deacetylated units. These parameters significantly influence its physic-chemical properties and are determined by the conditions set during alkaline deacetylation of chitin (Berger et al. 2004b, Elsabee and Abdou 2013, Berger et al. 2004a).

Chitosan is a weak base and is insoluble in water, but soluble in dilute aqueous acidic solutions below its pKa ( 6.3), in which it can convert glucosamine units due to the presence of amino groups $\left(-\mathrm{NH}_{2}\right)$ into the soluble protonated form $\left(-\mathrm{NH}_{3}{ }^{+}\right)$. Chitosan properties depend on its biological origin, molecular weight and degree of deacetylation. In fact, decreasing the polymer's degree of acetylation enhances the properties of chitosan since such properties are intrinsically related with the polymer's amino groups that are cationic in acidic-neutral media, like in physiological fluids (Goy et al. 2009, Leceta et al. 2013, Oliveira et al. 2012).

Currently, much attention is being paid to chitosan for its potential medical and pharmaceutical applications. A significant advantage of chitosan is its biocompatibility, which allows its use in various medical applications such as topical ocular application, implantation or injection. In addition, chitosan is considered as biodegradable since is metabolized by certain human enzymes, especially lysozyme. Due to its positive charge at physiological $\mathrm{pH}$, chitosan is also bioadhesive, which increases retention at the site of application. It has been also reported that chitosan acts as a penetration enhancer by opening epithelial tightjunctions (Berger et al. 2004b, Ravi Kumar 2000, Berger et al. 2004a, Bernkop-Schnürch and Dünnhaupt 2012).

Due to its ability to promote cell proliferation, naturally hydrophilic character, $\mathrm{pH}$-dependent cationic nature and tendency to interact with negatively charged glycosaminoglycans (GAG), heparin, proteoglycans, and polynucleotides like DNA or small interfering ribonucleic acid (siRNA), make chitosan particularly suitable as a biomaterial for tissue repair and regeneration. In this context, chitosan has been widely used as implants for controlled drug release and in scaffolds to deliver growth factors (Tan et al. 2014, Pandis et al. 2014, Oliveira et al. 2012, Gao et al. 2009). Chitosan has also been found to possess a growth-inhibitory effect on tumor cells, suggesting that chitosan may have potential value in cancer therapy (Tan et al. 2014). Furthermore, chitosan assembles many important requirements for biomedical applications, in that it promotes wound-healing, due to its ability to foster adequate granulation tissue formation accompanied by angiogenesis and regular deposition of thin collagen fibers. Finally, chitosan has both antimicrobial activity and bacteriostatic effects (Berger et al. 2004b, Tan et al. 2014, Muzzarelli 2009). The antimicrobial activity of 
chitosan was observed against a broad spectrum of microorganisms including fungi, algae and bacteria, being more active against Gram positive than Gram negative bacteria, in particular if it possess high molecular weight (Costa et al. 2012, Kong et al. 2010).

Moreover, the reactive functional groups present in chitosan, namely the amino group at the C2 position of each deacetylated unit and hydroxyl groups at the C6 and C3 positions, can be readily subjected to chemical modification to generate a series of chitosan derivatives with new mechanical and solubility properties, enlarged biocompatibility and tailored properties (Goy et al. 2009, Leceta et al. 2013, Zhang et al. 2012).

Interestingly, chitosan has the ability to be shaped in various forms such as films, microparticles, fibers and three-dimensional composite scaffolds for tissue engineering, all of them being largely used in different biomedical applications (Pandis et al. 2014, Ravi Kumar 2000, Rinaudo 2006, Yuan et al. 2010).

The properties of chitosan described above point out its great potential as a biofunctional material with promising results for tissue engineering and several other biomedical applications, such as drug and gene delivery (Croisier and Jérôme 2013, Carlson et al. 2008, Lieder et al. 2012, Costa et al. 2014, Shukla et al. 2013).

The principal characteristics of chitosan and related effects are summarized and displayed in the next table (Table 1).

Properties

Polycationic

Biocompatible

Biodegradable

Promotes woundhealing

Amino and alcoho functions along

chitosan chains

\section{Main observed effects}

Bioadhesive; interact with negatively charged GAG, heparin, proteoglycans, and polynucleotides like DNA or siRNA; antimicrobial activity; bacteriostatic effects and also analgesic effect

Used in various medical applications, such as topical ocular application, implantation or injection

Metabolized by certain human enzymes, especially lysozyme

Ability to foster adequate granulation tissue formation accompanied by angiogenesis and regular deposition of thin collagen fibers

Can be easily functionalized to further extend chitosan field of applications
References

Costa et al. 2012, Kong

et al. 2010

Bernkop-Schnürch and

Dünnhaupt 2012

Berger et al. 2004b

Costa et al. 2012, Kong

et al. 2010

Goy et al. 2009, Leceta et al. 2013, Zhang et al. 2012

Table 1: Chitosan properties that make it an attractive polymer for biomedical applications

\section{3. "Click" chemistry as a tool to create novel chitosan-based biomaterials}

\section{1 "Click" chemistry as a tethering tool}

"Click" chemistry reaction is a most promising, yet underexplored, approach which can be used for synthesis of various topological polymers or polymer modification (Iha et al. 2009, Wang et al. 2014). "Click" chemistry is a global term that refers to highly chemoselective and reactive reactions where two functional groups exclusively react with each other, even in the presence of other reactive functionalities. This bioorthogonal immobilization method has the ability to covalent assembly two chemically different molecules under mild conditions, such as complex carbohydrates with peptides, or chemoreporters, such as fluorescent dyes, with biopolymers. The reaction can be performed at room or physiological temperature and occurs in a manner that is high-yielding, rapid, modular, stereospecific, compatible with aqueous environments, and orthogonal to protecting and functional groups. So, the number of applications of "click" chemistry is growing in research areas, such as organic chemistry, bioconjugation, drug discovery, polymers, and radiochemistry (Nwe and Brechbiel 2009, Zeng 
et al. 2013, Amblard et al. 2009, Jiang et al. 2014, Nimmo and Shoichet 2011, Deng et al. 2011, Huerta-Angeles et al. 2012). Within the field of regenerative medicine "click" reactions have been used as bioconjugation techniques for decorating 2D cell substrates, and as promising patterning chemistries for immobilizing bioactive factors within 3D scaffolds (Nimmo and Shoichet 2011).

\subsubsection{Huisgen's 1,3-dipolar cycloaddition}

The Huisgen's 1,3-dipolar cycloaddition reaction between alkynes and azides yielding triazoles is the principal example of a "click" reaction and one of the most attractive chemoselective "click" reactions (Figure 1). Azides and alkynes can be easily incorporated into a wide range of substituents and are stable under a variety of conditions. Both functionalities are also essentially inert to most biological and organic conditions, molecular oxygen, water, and the majority of common reaction conditions in organic synthesis. The potential of this reaction is very high, since alkyne and azide components react selectively with each other (Meldal and Tornøe 2008, Kolb and Sharpless 2003, Tron et al. 2008, Jagasia et al. 2009, Agalave et al. 2011).

1,4-regioisomer

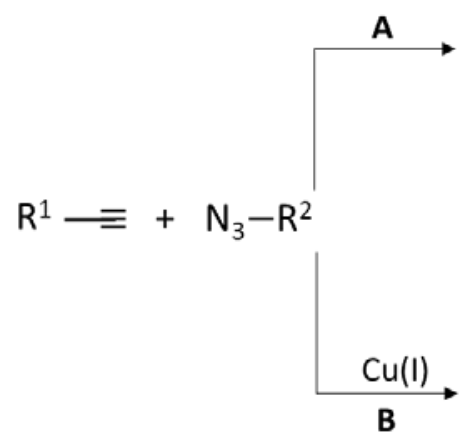<smiles>[R]c1cn([R])nn1</smiles>

$\mathrm{R}^{1}$<smiles>[R]c1cn([R])nn1</smiles>

1,5-regioisomer

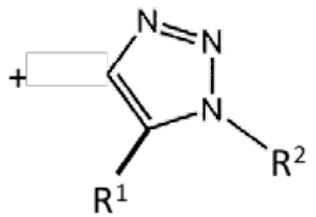

$\mathrm{R}^{1}$

Figure 1: The 1,3-dipolar cycloaddition between azides and alkynes: (A) unactivated azide-alkyne cycloaddition yielding a mixture of the 1,5- and the 1,4-triazole regioisomers and (B) copper (I) ( $\mathrm{Cu}(\mathrm{I}))$

catalysis in alkyne-azide coupling reactions favoring the formation of only the 1,4-regioisomer

The unactivated azide-alkyne cycloaddition was first discovered by Huisgen in 1963 and required high temperatures and pressures, since azides and alkynes have low reactivity towards each other at atmospheric pressure and room temperature. This stability, being purely kinetic in origin, is responsible for the slow nature of the cycloaddition reaction and the inertness of these functional groups towards biological molecules and towards the reaction conditions inside living systems (i.e. aqueous, and mild reducing environments). Furthermore, as the directing effect of the substituents is usually weak, this reaction suffered from lack of regioselectivity, yielding a mixture of the 1,4- and the 1,5-triazole regioisomers, at times laborious to separate using classical chromatographic procedures. These issues were overcome by Tornøe and Meldal, who introduced $\mathrm{Cu}(\mathrm{l})$ catalysis in alkyne-azide coupling reactions, which led to a major improvement in both rate and regioselectivity; the copper catalyst favors formation of only the 1,4-regioisomer (Nwe and Brechbiel 2009, Meldal and Tornøe 2008, Kolb and Sharpless 2003, Tron et al. 2008, Amblard et al. 2009, Tornøe et al. 2002b, Rostovtsev et al. 2002, Deng et al. 2011).

The formation of triazoles from azides and terminal alkynes catalyzed by $\mathrm{Cu}(\mathrm{I})$ is an extraordinarily robust reaction, which could be performed under a wide variety of conditions 
and with almost any source of solvated $\mathrm{Cu}(\mathrm{I})$. The most common conditions are the aqueous conditions employing copper sulfate $\left(\mathrm{CuSO}_{4}\right)$ (Meldal and Tornøe 2008). The standard catalytic system uses copper (II) salts (e.g., copper sulfate pentahydrate or copper acetate) in the presence of a reducing agent, such as sodium ascorbate or metallic copper. This constantly reduces copper (II) to $\mathrm{Cu}(\mathrm{I})$ maintaining significantly high levels of the catalytic species (Tron et al. 2008, Castro et al. 2012).

The triazole link created between the two building blocks (BB) that are coupled is very stable and chemically inert to most reactive conditions. In contrast to amides, and due to their high aromatic stabilization, triazoles cannot be cleaved hydrolytically or otherwise, and unlike benzenoids and related aromatic heterocycles, they are almost impossible to oxidize or reduce (Kolb and Sharpless 2003). These heterocycle have also an intermediate polarity with a high dipolar moment of $\sim 5 \mathrm{D}$ and are able to participate actively in hydrogen bond formation as well as in dipole-dipole and $\pi$ stacking interactions (Meldal and Tornøe 2008, Agalave et al. 2011, Schulze and Schubert 2014, Chen et al. 2007). Finally, triazoles are also relatively resistant to metabolic degradation (Tron et al. 2008, Agalave et al. 2011) and, interestingly, have been found to display diverse biological activities, including anti-HIV, antituberculosis and anticancer activities and also antimicrobial activity against Gram positive bacteria (Tornøe et al. 2002a, Agalave et al. 2011).

As the archetypal example of "click" chemistry, the $\mathrm{Cu}(\mathrm{I})$-catalyzed Huisgen 1,3-dipolar cycloaddition of azides and terminal alkynes (CuAAC) has been widely used in materials science and synthesis, molecular biology, and medicinal chemistry. Polysaccharides have been often modified using this reaction for different purposes such as creating new materials, new therapeutic or diagnostic agents, as a surface-modification strategy (Deng et al. 2011, Grischenko et al. 2015).

\subsection{Chitosan-based biomaterials}

Chitosan has been widely used as a drug delivery system, wound dressing, anticoagulant, and scaffold for tissue engineering owing to its properties. In recent years there has been a growing demand to broaden the applications of chitosan leading to the development of new techniques for enhancing its unique properties and develop multifunctional novel chitosan hybrid materials (Li et al. 2010, Bao et al. 2010b).

However, applications of chitosan are limited by its poor solubility in water and in common solvents, being only soluble in acidic aqueous solutions where the amino groups are protonated. In this context, graft modification takes place as an interesting method to overcome this limitation. Moreover, this technique has also been explored in the development of multifunctional chitosan copolymers with well-defined structures and enhanced properties for specific applications (Kulbokaite et al. 2009, Zhang et al. 2012, Zampano et al. 2010, Yuan et al. 2011, Munro et al. 2009).

In this context, efficient and high yielding "click" type reactions are useful and versatile tools. In particular, the CuAAC to give 1,4-regioselectively substituted triazoles has attracted much attention for the modification of polymers. Indeed bioconjugation, surface modification, and orthogonal functionalization of polymers were successfully performed. In recent literature the interest has been shown in this cycloaddition for the modification of polysaccharides, however, only a few chitosan graft copolymers have been synthesized by this technique (Zampano et al. 2010, Yuan et al. 2011).

Stimuli-responsive drug delivery systems are been extensively investigated in order to improve drug delivery to a target site and therapeutic efficacy while minimizing side effects. 
Biodegradable and biocompatible systems are been developed to respond to several stimuli, such as $\mathrm{pH}$, temperature, redox or light (Guerry et al. 2014). Several polymers were already grafted to the chitosan backbone for the development of stimuli-responsive polymers. These polymers have attracted considerable interest for several biomedical applications such as smart bioactive surfaces, selective bioseparation, affinity control, or drug delivery. Poly(Nisopropylacrylamide) (PNIPAM) and poly[(2-dimethylamino)ethyl methacrylate] (PDMAEMA) were successfully grafted onto chitosan providing both temperature and $\mathrm{pH}$ responsive copolymer (Bao et al. 2010b, Bao et al. 2010a).

Yuan and co-workers developed, via "click" chemistry, a novel amphiphilic chitosan graft copolymer that could assemble into spherical micelles with termosensitive properties (Yuan et al. 2011). Micellar drug delivery systems have been widely investigated due to prolonged circulation in the blood, improved stability, and enhanced accumulation in tissues. Results showed that the chitosan graft copolymer micelles are promising candidates as intelligent carriers in controlled drug release system (Yuan et al. 2011, Wu et al. 2009).

According to the work of Zampano et al. selective functionalization and crosslinking of chitosan by azide-alkyne dipolar cycloaddition, showed to be particularly promising for the development of chitosan-based gels with $\mathrm{pH}$-dependent swellability properties (Zampano et al. 2010, Uliniuc et al. 2012).

A wide variety of biodegradable redox-sensitive amphiphilic copolymers has been described to incorporate and release drugs. In this context, amphiphilic chitosan-based graft copolymers containing a chitosan backbone and hydrophobic pendent blocks have been extensively studied (Guerry et al. 2014). "Click" reaction was used to synthesize amphiphilic graft copolymers composed of poly( $\varepsilon$-caprolactone) and chitosan. Their self-assembly in water, followed by core-cross-clicking with bis-alkynyl cystamine, led to redox-sensitive drug release nanoparticles. These drug-loaded cross-linked nanoparticles were effectively decross-linked in reductive environments mimicking cytoplasm and cell nucleus ones to release doxorubicin, an anticancer drug (Guerry et al. 2014).

Bertoldo and colleagues reported the synthesis of a new light responsive polymer through CUAAC. Spiropyrans are a class of organic compounds with the ability to vary their chemical structure when exposed to light of an appropriate wavelength, and regain their original isomer when placed in the dark, or upon exposure to light of a different wavelength. The reaction between a 6-azido-6-deoxy, N-phthaloyl derivative of chitosan and a photochromic spiropyran resulted in a new chitosan based material with an interesting photochromic response to light irradiation (Bertoldo et al. 2011).

A highly regioselective amphiprotic chitosan derivative, which can either donate or accept protons, was synthesized via "click" chemistry using 6-azido-6-deoxy chitosan derivative. The structure with positive $\left(-\mathrm{NH}_{3}{ }^{+}\right)$and negative $\left(-\mathrm{COO}^{-}\right)$ions made the compound soluble under both basic and acidic conditions, respectively. Furthermore, it had the ability to form nanoparticles in neutral aqueous solution due to the inter- and intra-molecular aggregation. These properties make it possible to be used as drug delivery systems (Ifuku et al. 2013).

CuACC was also applied to graft methoxy poly(ethylene glycol) (MPEG) onto N-azidated chitosan. PEGylation of polymers is one of the strategies used to overcome the uptake of polymer micelles by macrophage which is still a challenge for clinical applications since it lead to reduced half-time of the carrier in blood. PEG is a nontoxic and nonirritant hydrophilic polymer with the potential to sterically stabilize nanoparticles against protein absorption and subsequent phagocytosis thus improving the stability of the drug delivery system. This 
approach allowed obtaining chitosan copolymers with predetermined composition and simplified the purification process (Kulbokaite et al. 2009, Hu et al. 2008, Lin et al. 2011).

In recent years, there has been a growing interest in the chemical modification of naturally occurring polysaccharides for developing non-viral gene vectors (Pang et al. 2015). Chitosan has been widely investigated for its cationic behavior and ability to bind to negatively charged molecules such as DNA, RNA, and oligonucleotide. Moreover, its low cytotoxicity has been demonstrated both in vivo studies and clinical trials. However, it is necessary to overcome the solubility limitation of chitosan and its low transfection efficiency. One of the routes to improve solubility is the regioselective modification of chitosan. Taking advantage of the concept of "click" chemistry, Gao and co-workers reported chitosan modification with a trimethylammonium cationic group through its hydroxyl groups in the $\mathrm{C} 6$ position. A non-toxic chitosan derivative was developed with increased DNA binding ability and cellular uptake suggesting that this new derivative could be an efficiently used for gene delivery (Gao et al. 2009, Zhao et al. 2013).

CuAAC "click" reaction can also been used in the fabrication of layer-by-layer (LbL) ultrathin polymeric films and subsequent formation of microcapsules (De Geest et al. 2008, Such et al. 2007). In the work of Zhang and colleagues this technique was used in the fabrication of chitosan microcapsules using LbL assembly. Moreover, this method allows postfunctionalization of chitosan with other materials, such as biomacromolecules, peptides or other functional groups thus creating microcapsules with additional properties, such as cellsspecific binding sites (Zhang et al. 2011).

Tirino et al. synthesized a chitosan-based hydrogel by means of "click" chemistry with improved mechanical properties. Polyethyleneoxide (PEO) was selected owning to its biocompatibility, $\mathrm{pH}$ independent swelling and drug release characteristics. The synthesized hydrogel appeared to be suitable for several applications, such as drug release and wound healing due to its controllable crosslinking degree and ability to rapidly absorb high amounts of water (Tirino et al. 2014).

Ryu and co-workers applied the CuCAA to the covalent functionalization graphene oxide (GO) sheets with chitosan. The functionalized graphene films displayed superior mechanical and highly enhanced antimicrobial properties make them competitive candidates for various biological applications (Ryu et al. 2013).

Table 2 presents an overview of the different chitosan derivatives obtained through CuAAC "click" reaction.

\begin{tabular}{|c|c|c|}
\hline Chitosan derivative & Advantages & References \\
\hline $\begin{array}{l}\text { Temperature and } \mathrm{pH} \text { responsive } \\
\text { copolymer composed of PNIPAM, } \\
\text { PDMAEMA and chitosan }\end{array}$ & $\begin{array}{l}\text { Stimuli-responsive drug delivery } \\
\text { systems }\end{array}$ & $\begin{array}{l}\text { Bao et al. 2010b, Bao } \\
\text { et al. 2010a }\end{array}$ \\
\hline $\begin{array}{l}\text { Amphiphilic graft copolymers composed } \\
\text { of poly( } \varepsilon \text {-caprolactone) and chitosan }\end{array}$ & $\begin{array}{c}\text { Redox-sensitive drug } \\
\text { nanoparticles to release an } \\
\text { anticancer drug }\end{array}$ & Guerry et al. 2014 \\
\hline $\begin{array}{l}\text { Regioselective amphiprotic 6-azido-6- } \\
\text { deoxy chitosan derivative }\end{array}$ & $\begin{array}{l}\text { Nanoparticles to be used as drug } \\
\text { delivery systems (Ifuku et al. } \\
\text { 2013) }\end{array}$ & Ifuku et al. 2013 \\
\hline PEGylation of chitosan & $\begin{array}{l}\text { Overcome the uptake of polymer } \\
\text { micelles by macrophage }\end{array}$ & Kulbokaite et al. 2009 \\
\hline $\begin{array}{l}\text { Chitosan microcapsules using LbL } \\
\text { assembly }\end{array}$ & $\begin{array}{l}\text { Chitosan microcapsules for use in } \\
\text { targeted drug delivery systems }\end{array}$ & Zhang et al. 2011 \\
\hline $\begin{array}{l}\text { Chitosan-based hydrogel functionalized } \\
\text { with PEO }\end{array}$ & $\begin{array}{l}\text { Improved mechanical properties } \\
\text { suitable for several applications, }\end{array}$ & Tirino et al. 2014 \\
\hline
\end{tabular}


Covalent functionalization graphene oxide (GO) sheets with chitosan such as drug release and wound healing

Superior mechanical and highly enhanced antimicrobial properties

Table 2: Functionalization strategies employing CuACC reaction to modify chitosan for different biomedical applications

The CuACC reaction allows the design of various chitosan based materials with controlled and well-defined structures. Besides, this synthetic strategy enables further chemical modification at the residual positions of chitosan, which leads to the creation of high performance chitosan derivatives with the possibility of having several different functional groups at each position (Ifuku et al. 2012, 2011).

\section{Conclusions}

Biological macromolecules, such as polymers, proteins and peptides, have been employed as indispensable biomaterials for decades. Polymers, in particular chitosan has been highlighted as a promising material due to its superior properties such as biocompatibility, chemical activity, abundance and relative low cost (Zhang and Cao 2014).

The "click" chemistry concept has emerged as a unique and versatile family of highly efficient organic reactions for the synthesis of bio-inspired biomaterials that can be tailored according its final applications such as controlled drug release, gene delivery, tissue engineering and regenerative medicine. This chemical approach has become a powerful method within combinatorial chemistry due to its high efficiency, specificity, mild reaction conditions and tolerance to most functional groups. Within the "click" chemistry toolbox, CuAAC proved to be a highly versatile and useful reaction leading to the 1,2,3-triazole heterocycle (Loaiza et al. 2009, Deng et al. 2014, Nimmo and Shoichet 2011).

As discussed in this review the CuACC reaction provides an attractive synthetic rout for welldefined biologically active chitosan-based moieties with specific properties. The synthesized polymers displayed different architectures namely block and graft polymers, films or hydrogels, tailored for each application. Over the past decade, this reaction has become a powerful approach within the biomedical and pharmaceutical fields to build materials that are cell compatible, highly functional and organized in structure (Azagarsamy and Anseth 2012, Chen et al. 2007, van Dijk et al. 2009).

Bioorthogonal "click" chemistry has gained a significant impact in biomaterials design and synthesis over the last years, however, it is still an open field for researchers interested in biomaterials and their applications and it is expected that its importance will increase in the coming years (Azagarsamy and Anseth 2012, van Dijk et al. 2009, Nimmo and Shoichet 2011).

\section{References}

Agalave, Sandip G., Suleman R. Maujan, and Vandana S. Pore. 2011. "Click Chemistry: 1,2,3Triazoles as Pharmacophores." Chemistry - An Asian Journal 6 (10):2696-2718. DOI: 10.1002/asia.201100432.

Amblard, Franck, Jong Hyun Cho, and Raymond F Schinazi. 2009. "Cu (I)-catalyzed Huisgen azide- alkyne 1, 3-dipolar cycloaddition reaction in nucleoside, nucleotide, and oligonucleotide chemistry." Chemical reviews 109 (9):4207-4220.

Azagarsamy, Malar A., and Kristi S. Anseth. 2012. "Bioorthogonal Click Chemistry: An Indispensable Tool to Create Multifaceted Cell Culture Scaffolds." ACS Macro Letters 2 (1):5-9. DOI: 10.1021/mz300585q. 
Bao, Hongqian, Lin Li, Leong Huat Gan, Yuan Ping, Jun Li, and Palaniswamy Ravi. 2010a. "Thermo- and pH-Responsive Association Behavior of Dual Hydrophilic Graft Chitosan Terpolymer Synthesized via ATRP and Click Chemistry." Macromolecules 43 (13):56795687. DOI: 10.1021/ma100894p.

Bao, Hongqian, Lin Li, Wai Chong Leong, and Leong Huat Gan. 2010b. "Thermo-Responsive Association of Chitosan-graft-Poly(N-isopropylacrylamide) in Aqueous Solutions." The Journal of Physical Chemistry B 114 (32):10666-10673. DOI: 10.1021/jp105041z.

Berger, J., M. Reist, J. M. Mayer, O. Felt, and R. Gurny. 2004a. "Structure and interactions in chitosan hydrogels formed by complexation or aggregation for biomedical applications." European Journal of Pharmaceutics and Biopharmaceutics 57 (1):35-52. DOI: 10.1016/S0939-6411(03)00160-7.

Berger, J., M. Reist, J. M. Mayer, O. Felt, N. A. Peppas, and R. Gurny. 2004b. "Structure and interactions in covalently and ionically crosslinked chitosan hydrogels for biomedical applications." European Journal of Pharmaceutics and Biopharmaceutics 57 (1):19-34. DOI: 10.1016/S0939-6411(03)00161-9.

Bernkop-Schnürch, Andreas, and Sarah Dünnhaupt. 2012. "Chitosan-based drug delivery systems." European Journal of Pharmaceutics and Biopharmaceutics 81 (3):463-469. DOI: 10.1016/j.ejpb.2012.04.007.

Bertoldo, Monica, Samuele Nazzi, Giovanni Zampano, and Francesco Ciardelli. 2011. "Synthesis and photochromic response of a new precisely functionalized chitosan with "clicked" spiropyran." Carbohydrate Polymers 85 (2):401-407. DOI: 10.1016/j.carbpol.2011.02.043.

Carlson, Ross P., Reed Taffs, William M. Davison, and Philip S. Stewart. 2008. "Anti-biofilm properties of chitosan-coated surfaces." Journal of Biomaterials Science, Polymer Edition 19 (8):1035-1046. DOI: 10.1163/156856208784909372.

Castro, Vida, Hortensia Rodriguez, and Fernando Albericio. 2012. "Wang Linker Free of Side Reactions." Organic Letters 15 (2):246-249. DOI: 10.1021/ol303367s.

Chen, Hao, Janet L. Taylor, and Suzanne R. Abrams. 2007. "Design and synthesis of $\beta$ methoxyacrylate analogues via click chemistry and biological evaluations." Bioorganic \& Medicinal Chemistry Letters 17 (7):1979-1983. DOI: 10.1016/j.bmcl.2007.01.021.

Costa, E. M., S. Silva, C. Pina, F. K. Tavaria, and M. M. Pintado. 2012. "Evaluation and insights into chitosan antimicrobial activity against anaerobic oral pathogens." Anaerobe 18 (3):305-309. DOI: 10.1016/j.anaerobe.2012.04.009.

Costa, Fabíola, Sílvia Maia, Joana Gomes, Paula Gomes, and M. Cristina L. Martins. 2014. "Characterization of hLF1-11 immobilization onto chitosan ultrathin films, and its effects on antimicrobial activity." Acta Biomaterialia 10 (8):3513-3521. DOI: 10.1016/j.actbio.2014.02.028.

Croisier, Florence, and Christine Jérôme. 2013. "Chitosan-based biomaterials for tissue engineering." European Polymer Journal 49 (4):780-792. DOI: 10.1016/j.eurpolymj.2012.12.009.

De Geest, Bruno G., Wim Van Camp, Filip E. Du Prez, Stefaan C. De Smedt, Jo Demeester, and Wim E. Hennink. 2008. "Degradable Multilayer Films and Hollow Capsules via a 'Click' Strategy." Macromolecular Rapid Communications 29 (12-13):1111-1118. DOI: 10.1002/marc.200800093.

Deng, Chao, Jintian Wu, Ru Cheng, Fenghua Meng, Harm-Anton Klok, and Zhiyuan Zhong. 2014. "Functional polypeptide and hybrid materials: Precision synthesis via $\alpha$-amino acid 
$\mathrm{N}$-carboxyanhydride polymerization and emerging biomedical applications." Progress in Polymer Science 39 (2):330-364. DOI: 10.1016/j.progpolymsci.2013.10.008.

Deng, Xiaopei, Christian Friedmann, and Joerg Lahann. 2011. "Bio-orthogonal "Double-Click" Chemistry Based on Multifunctional Coatings." Angewandte Chemie International Edition 50 (29):6522-6526. DOI: 10.1002/anie.201101581.

Elsabee, Maher Z., and Entsar S. Abdou. 2013. "Chitosan based edible films and coatings: A review." Materials Science and Engineering: C 33 (4):1819-1841. DOI: 10.1016/j.msec.2013.01.010.

Gao, Yu, Zhiwen Zhang, Lingli Chen, Wangwen Gu, and Yaping Li. 2009. "Synthesis of 6-N,N,NTrimethyltriazole Chitosan via "Click Chemistry" and Evaluation for Gene Delivery." Biomacromolecules 10 (8):2175-2182. DOI: 10.1021/bm900341d.

Goy, R. C., D. De Britto, and O. B. G. Assis. 2009. "A review of the antimicrobial activity of chitosan." Polimeros 19 (3):241-247.

Grischenko, Lyudmila A., Lidiya N. Parshina, Lyudmila I. Larina, Lyubov N. Novikova, and Boris A. Trofimov. 2015. "Modification of arabinogalactan propargyl ethers by triazolyl functions." Carbohydrate Polymers 115 (0):294-304. DOI: 10.1016/j.carbpol.2014.08.050.

Guerry, Alexandre, Sylvain Cottaz, Etienne Fleury, Julien Bernard, and Sami Halila. 2014. "Redox-stimuli responsive micelles from DOX-encapsulating polycaprolactone-g-chitosan oligosaccharide." Carbohydrate Polymers 112 (0):746-752. DOI: 10.1016/j.carbpol.2014.06.052.

Hu, Fu-Qiang, Pan Meng, You-Qin Dai, Yong-Zhong Du, Jian You, Xiao-Hong Wei, and Hong Yuan. 2008. "PEGylated chitosan-based polymer micelle as an intracellular delivery carrier for anti-tumor targeting therapy." European Journal of Pharmaceutics and Biopharmaceutics 70 (3):749-757. DOI: 10.1016/j.ejpb.2008.06.015.

Huerta-Angeles, Gloria, Miroslava Němcová, Eva Př́kopová, Daniela Šmejkalová, Martin Pravda, Lukáš Kučera, and Vladimír Velebný. 2012. "Reductive alkylation of hyaluronic acid for the synthesis of biocompatible hydrogels by click chemistry." Carbohydrate Polymers 90 (4):1704-1711. DOI: 10.1016/j.carbpol.2012.07.054.

Ifuku, Shinsuke, Chisato Matsumoto, Masahiro Wada, Minoru Morimoto, and Hiroyuki Saimoto. 2013. "Preparation of highly regioselective amphiprotic chitosan derivative via "click chemistry"." International Journal of Biological Macromolecules 52 (0):72-76. DOI: 10.1016/j.ijbiomac.2012.08.012.

Ifuku, Shinsuke, Masahiro Wada, Minoru Morimoto, and Hiroyuki Saimoto. 2011. "Preparation of highly regioselective chitosan derivatives via "click chemistry"." Carbohydrate Polymers 85 (3):653-657. DOI: 10.1016/j.carbpol.2011.03.030.

Ifuku, Shinsuke, Masahiro Wada, Minoru Morimoto, and Hiroyuki Saimoto. 2012. "A short synthesis of highly soluble chemoselective chitosan derivatives via "click chemistry"." Carbohydrate Polymers 90 (2):1182-1186. DOI: 10.1016/j.carbpol.2012.06.074.

Iha, Rhiannon K., Karen L. Wooley, Andreas M. Nyström, Daniel J. Burke, Matthew J. Kade, and Craig J. Hawker. 2009. "Applications of Orthogonal "Click" Chemistries in the Synthesis of Functional Soft Materials." Chemical Reviews 109 (11):5620-5686. DOI: 10.1021/cr900138t.

Jagasia, Reshma, Justin M Holub, Markus Bollinger, Kent Kirshenbaum, and MG Finn. 2009. "Peptide Cyclization and Cyclodimerization by Cul-Mediated Azide- Alkyne Cycloaddition." The Journal of organic chemistry 74 (8):2964-2974. 
Jiang, Yanjiao, Jing Chen, Chao Deng, Erik J. Suuronen, and Zhiyuan Zhong. 2014. "Click hydrogels, microgels and nanogels: Emerging platforms for drug delivery and tissue engineering." Biomaterials 35 (18):4969-4985. DOI: 10.1016/j.biomaterials.2014.03.001.

Kolb, Hartmuth C, and K Barry Sharpless. 2003. "The growing impact of click chemistry on drug discovery." Drug discovery today 8 (24):1128-1137.

Kolb, Hartmuth C., M. G. Finn, and K. Barry Sharpless. 2001. "Click Chemistry: Diverse Chemical Function from a Few Good Reactions." Angewandte Chemie International Edition 40 (11):2004-2021. DOI: 10.1002/1521-3773(20010601)40:11<2004::AID-ANIE2004>3.0.CO;2-5.

Kong, Ming, Xi Guang Chen, Ke Xing, and Hyun Jin Park. 2010. "Antimicrobial properties of chitosan and mode of action: A state of the art review." International Journal of Food Microbiology 144 (1):51-63. DOI: 10.1016/j.ijfoodmicro.2010.09.012.

Kulbokaite, Ruta, Gediminas Ciuta, Milos Netopilik, and Ricardas Makuska. 2009. "NPEG'ylation of chitosan via "click chemistry" reactions." Reactive and Functional Polymers 69 (10):771-778. DOI: 10.1016/j.reactfunctpolym.2009.06.010.

Leceta, I, P Guerrero, and K de la Caba. 2013. "Functional properties of chitosan-based films." Carbohydrate polymers 93 (1):339-346.

Li, Xiaofei, Weizhong Yuan, Shuying Gu, and Jie Ren. 2010. "Synthesis and self-assembly of tunable thermosensitive chitosan amphiphilic copolymers by click chemistry." Materials Letters 64 (24):2663-2666. DOI: 10.1016/j.matlet.2010.08.077.

Lieder, Ramona, Mariam Darai, Margrét Björk Thor, C. H. Ng, Jón M. Einarsson, Sveinn Gudmundsson, Benedikt Helgason, Vivek Sambhaji Gaware, Már Másson, Jóhannes Gíslason, Gissur Örlygsson, and Ólafur E. Sigurjónsson. 2012. "In vitro bioactivity of different degree of deacetylation chitosan, a potential coating material for titanium implants." Journal of Biomedical Materials Research Part A 100A (12):3392-3399. DOI: 10.1002/jbm.a.34283.

Lin, Wen Jen, Tze Dan Chen, Chia Wen Liu, Jiin Long Chen, and Fu Hsiung Chang. 2011. "Synthesis of lactobionic acid-grafted-pegylated-chitosan with enhanced HepG2 cells transfection." Carbohydrate Polymers 83 (2):898-904. DOI: 10.1016/j.carbpol.2010.08.072.

Loaiza, Pilar Rodriguez, Stefan Löber, Harald Hübner, and Peter Gmeiner. 2009. "Parallel synthesis of potent dopaminergic $\mathrm{N}$-phenyltriazole carboxamides applying a novel click chemistry based phenol linker." Bioorganic \& Medicinal Chemistry 17 (15):5482-5487. DOI: 10.1016/j.bmc.2009.06.041.

Meldal, Morten, and Christian Wenzel Tornøe. 2008. "Cu-catalyzed azide- alkyne cycloaddition." Chemical reviews 108 (8):2952-3015.

Munro, Natasha H., Lyall R. Hanton, Stephen C. Moratti, and Brian H. Robinson. 2009. "Synthesis and characterisation of chitosan-graft-poly(OEGMA) copolymers prepared by ATRP." Carbohydrate Polymers 77 (3):496-505. DOI: 10.1016/j.carbpol.2009.01.026.

Muzzarelli, Riccardo A. A. 2009. "Chitins and chitosans for the repair of wounded skin, nerve, cartilage and bone." Carbohydrate Polymers 76 (2):167-182. DOI: 10.1016/j.carbpol.2008.11.002.

Nimmo, Chelsea M., and Molly S. Shoichet. 2011. "Regenerative Biomaterials that "Click": Simple, Aqueous-Based Protocols for Hydrogel Synthesis, Surface Immobilization, and 3D Patterning." Bioconjugate Chemistry 22 (11):2199-2209. DOI: 10.1021/bc200281k.

Nwe, Kido, and Martin W Brechbiel. 2009. "Growing applications of "click chemistry" for bioconjugation in contemporary biomedical research." Cancer Biotherapy and Radiopharmaceuticals 24 (3):289-302. 
Oliveira, José Ricardo, M. Cristina L. Martins, Luís Mafra, and Paula Gomes. 2012. "Synthesis of an O-alkynyl-chitosan and its chemoselective conjugation with a PEG-like amino-azide through click chemistry." Carbohydrate Polymers 87 (1):240-249. DOI: 10.1016/j.carbpol.2011.07.043.

Pandis, Christos, Sara Madeira, Joana Matos, Apostolos Kyritsis, João F. Mano, and José Luis Gómez Ribelles. 2014. "Chitosan-silica hybrid porous membranes." Materials Science and Engineering: C 42 (0):553-561. DOI: 10.1016/j.msec.2014.05.073.

Pang, Jia-Dong, Bao-Xiong Zhuang, Kaijin Mai, Ru-Fu Chen, Jie Wang, and Li-Ming Zhang. 2015. "Click modification of helical amylose by poly(l-lysine) dendrons for non-viral gene delivery." Materials Science and Engineering: C 49 (0):485-492. DOI: 10.1016/j.msec.2015.01.011.

Ravi Kumar, Majeti N. V. 2000. "A review of chitin and chitosan applications." Reactive and Functional Polymers 46 (1):1-27. DOI: 10.1016/S1381-5148(00)00038-9.

Rinaudo, Marguerite. 2006. "Chitin and chitosan: Properties and applications." Progress in Polymer Science 31 (7):603-632. DOI: 10.1016/j.progpolymsci.2006.06.001.

Rostovtsev, Vsevolod V., Luke G. Green, Valery V. Fokin, and K. Barry Sharpless. 2002. "A Stepwise Huisgen Cycloaddition Process: Copper(I)-Catalyzed Regioselective "Ligation" of Azides and Terminal Alkynes." Angewandte Chemie International Edition 41 (14):25962599. DOI: 10.1002/1521-3773(20020715)41:14<2596::AID-ANIE2596>3.0.CO;2-4.

Ryu, Hee Jeong, Sibdas Singha Mahapatra, Santosh Kumar Yadav, and Jae Whan Cho. 2013. "Synthesis of click-coupled graphene sheet with chitosan: Effective exfoliation and enhanced properties of their nanocomposites." European Polymer Journal 49 (9):26272634. DOI: 10.1016/j.eurpolymj.2013.06.005.

Schulze, Benjamin, and Ulrich S. Schubert. 2014. "Beyond click chemistry - supramolecular interactions of 1,2,3-triazoles." Chemical Society Reviews 43 (8):2522-2571. DOI: 10.1039/C3CS60386E.

Shukla, Sudheesh K., Ajay K. Mishra, Omotayo A. Arotiba, and Bhekie B. Mamba. 2013. "Chitosan-based nanomaterials: A state-of-the-art review." International Journal of Biological Macromolecules 59 (0):46-58. DOI: 10.1016/j.ijbiomac.2013.04.043.

Such, Georgina K., Elvira Tjipto, Almar Postma, Angus P. R. Johnston, and Frank Caruso. 2007. "Ultrathin, Responsive Polymer Click Capsules." Nano Letters 7 (6):1706-1710. DOI: 10.1021/nl070698f.

Tan, Mei L., Peng Shao, Anna M. Friedhuber, Mallory van Moorst, Mina Elahy, Sivanjah Indumathy, Dave E. Dunstan, Yongzhong Wei, and Crispin R. Dass. 2014. "The potential role of free chitosan in bone trauma and bone cancer management." Biomaterials 35 (27):78287838. DOI: 10.1016/j.biomaterials.2014.05.087.

Tirino, Pasquale, Rosaria Laurino, Giovanni Maglio, Mario Malinconico, Giovanna Gomez d'Ayala, and Paola Laurienzo. 2014. "Synthesis of chitosan-PEO hydrogels via mesylation and regioselective $\mathrm{Cu}(\mathrm{I})$-catalyzed cycloaddition." Carbohydrate Polymers 112 (0):736-745. DOI: 10.1016/j.carbpol.2014.06.026.

Tornøe, Christian W, Caspar Christensen, and Morten Meldal. 2002a. "Peptidotriazoles on solid phase:[1, 2, 3]-triazoles by regiospecific copper (I)-catalyzed 1, 3-dipolar cycloadditions of terminal alkynes to azides." The Journal of organic chemistry 67 (9):30573064.

Tornøe, Christian W., Caspar Christensen, and Morten Meldal. 2002b. "Peptidotriazoles on Solid Phase: [1,2,3]-Triazoles by Regiospecific Copper(I)-Catalyzed 1,3-Dipolar 
Cycloadditions of Terminal Alkynes to Azides." The Journal of Organic Chemistry 67 (9):3057-3064. DOI: 10.1021/jo011148j.

Tron, Gian Cesare, Tracey Pirali, Richard A Billington, Pier Luigi Canonico, Giovanni Sorba, and Armando A Genazzani. 2008. "Click chemistry reactions in medicinal chemistry: Applications of the 1, 3-dipolar cycloaddition between azides and alkynes." Medicinal research reviews 28 (2):278-308.

Uliniuc, A, M Popa, Thierry Hamaide, and M Dobromir. 2012. "New approaches in hydrogel synthesis-Click chemistry: A review." Cellulose Chemistry and Technology 46 (1):1.

van Dijk, Maarten, Dirk T. S. Rijkers, Rob M. J. Liskamp, Cornelus F. van Nostrum, and Wim E. Hennink. 2009. "Synthesis and Applications of Biomedical and Pharmaceutical Polymers via Click Chemistry Methodologies." Bioconjugate Chemistry 20 (11):2001-2016. DOI: 10.1021/bc900087a.

Wang, Shuangshuang, Xiaoke Yang, Wen Zhu, Lei Zou, Ke Zhang, Yongming Chen, and Fu Xi. 2014. "Strain-promoted azide-alkyne cycloaddition "click" as a conjugation tool for building topological polymers." Polymer 55 (19):4812-4819. DOI: 10.1016/j.polymer.2014.08.003.

Wu, De-Qun, Bo Lu, Cong Chang, Chang-Sheng Chen, Tao Wang, Yuan-Yuan Zhang, Si-Xue Cheng, Xue-Jun Jiang, Xian-Zheng Zhang, and Ren-Xi Zhuo. 2009. "Galactosylated fluorescent labeled micelles as a liver targeting drug carrier." Biomaterials 30 (7):13631371. DOI: 10.1016/j.biomaterials.2008.11.027.

Yuan, Q., J. Shah, S. Hein, and R. D. K. Misra. 2010. "Controlled and extended drug release behavior of chitosan-based nanoparticle carrier." Acta Biomaterialia 6 (3):1140-1148. DOI: 10.1016/j.actbio.2009.08.027.

Yuan, Weizhong, Xiaofei Li, Shuying Gu, Amin Cao, and Jie Ren. 2011. "Amphiphilic chitosan graft copolymer via combination of ROP, ATRP and click chemistry: Synthesis, selfassembly, thermosensitivity, fluorescence, and controlled drug release." Polymer 52 (3):658-666.

Zampano, Giovanni, Monica Bertoldo, and Francesco Ciardelli. 2010. "Defined Chitosan-based networks by C-6-Azide-alkyne "click" reaction." Reactive and Functional Polymers 70 (5):272-281. DOI: 10.1016/j.reactfunctpolym.2010.01.004.

Zeng, Dexing, Brian M Zeglis, Jason S Lewis, and Carolyn J Anderson. 2013. "The growing impact of bioorthogonal click chemistry on the development of radiopharmaceuticals." Journal of Nuclear Medicine 54 (6):829-832.

Zhang, Jing, Cao Li, Zhi-Yuan Xue, Hai-Wei Cheng, Fu-Wei Huang, Ren-Xi Zhuo, and Xian-Zheng Zhang. 2011. "Fabrication of lactobionic-loaded chitosan microcapsules as potential drug carriers targeting the liver." Acta biomaterialia 7 (4):1665-1673.

Zhang, Ke, Pengyu Zhuang, Zhengke Wang, Youliang Li, Zhiqiang Jiang, Qiaoling Hu, Minying Liu, and Qingxiang Zhao. 2012. "One-pot synthesis of chitosan-g-(PEO-PLLA-PEO) via "click" chemistry and "SET-NRC" reaction." Carbohydrate Polymers 90 (4):1515-1521. DOI: 10.1016/j.carbpol.2012.07.023.

Zhang, Ping, and Moyuan Cao. 2014. "Preparation of a novel organo-soluble chitosan grafted polycaprolactone copolymer for drug delivery." International Journal of Biological Macromolecules 65 (0):21-27. DOI: 10.1016/j.ijbiomac.2014.01.009.

Zhao, Xiaoli, Zhaoyang Li, Haobo Pan, Wenguang Liu, Minmin Lv, Frankie Leung, and William W. Lu. 2013. "Enhanced gene delivery by chitosan-disulfide-conjugated LMW-PEI for facilitating osteogenic differentiation." Acta Biomaterialia 9 (5):6694-6703. DOI: 10.1016/j.actbio.2013.01.039. 